\title{
Die Neubildung Sachsens 1989/1990 und der Festakt am 3. Oktober 1990 auf der Albrechtsburg in Meißen
}

\author{
Michael Richter
}

Am 3. Oktober 1990 wurde auf der Albrechtsburg zu Meißen die Neubildung Sachsens als Freistaat der Bundesrepublik Deutschland festlich begangen. Der Ort war mit Bedacht gewählt. Die Errichtung der Burg Meißen im Jahre 929 war der Ausgangspunkt der Jahrhunderte währenden sächsischen Landesgeschichte. Was lag näher, als nach Jahrzehnten erzwungener sächsischer Nichtstaatlichkeit an diesem Ort den auch in der DDR nie ganz fallengelassenen Faden sächsischer Geschichte wieder aufzunehmen? Möglich wurde dies dadurch, dass auch und vielleicht vor allem die Sachsen in der friedlichen Revolution ihre Forderungen nach Freiheit, Wohlstand und deutscher Einheit mit der nach Rückkehr zur alten Länderstruktur verbanden.

Seit dem Herbst 1989 gab es in Sachsen Forderungen nach der Wiedereinführung der Länderstruktur. Bereits im November wurden Sachsen-Transparente auf allen wichtigen Demonstrationen mitgeführt, und die Forderung nach der Bildung von Ländern wurde eine wichtige Triebkraft der revolutionären Entwicklung. Dabei waren auch Meißen und Nachbarkommunen wie Coswig oder Radebeul wichtige Zentren des revolutionären Geschehens. In Dresden setzten sich Mitglieder der Gruppe der 20 bereits am 23. November für die Wiedererrichtung der Länder ein und begannen wenig später mit der Ausarbeitung erster Entwürfe einer sächsischen Verfassung. Spätestens seit dem Besuch von Bundeskanzler Helmut Kohl in Dresden am 19. Dezember dominierten weiß-grüne Sachsenfahnen neben Deutschlandflaggen das Bild der Demonstrationen. Die Forderung nach Bildung des Landes Sachsen wurde von einer großen Mehrheit der Bevölkerung getragen. Die Landesfarben an Wohnhäusern und Straßenrändern sowie Sachsen-Aufkleber auf

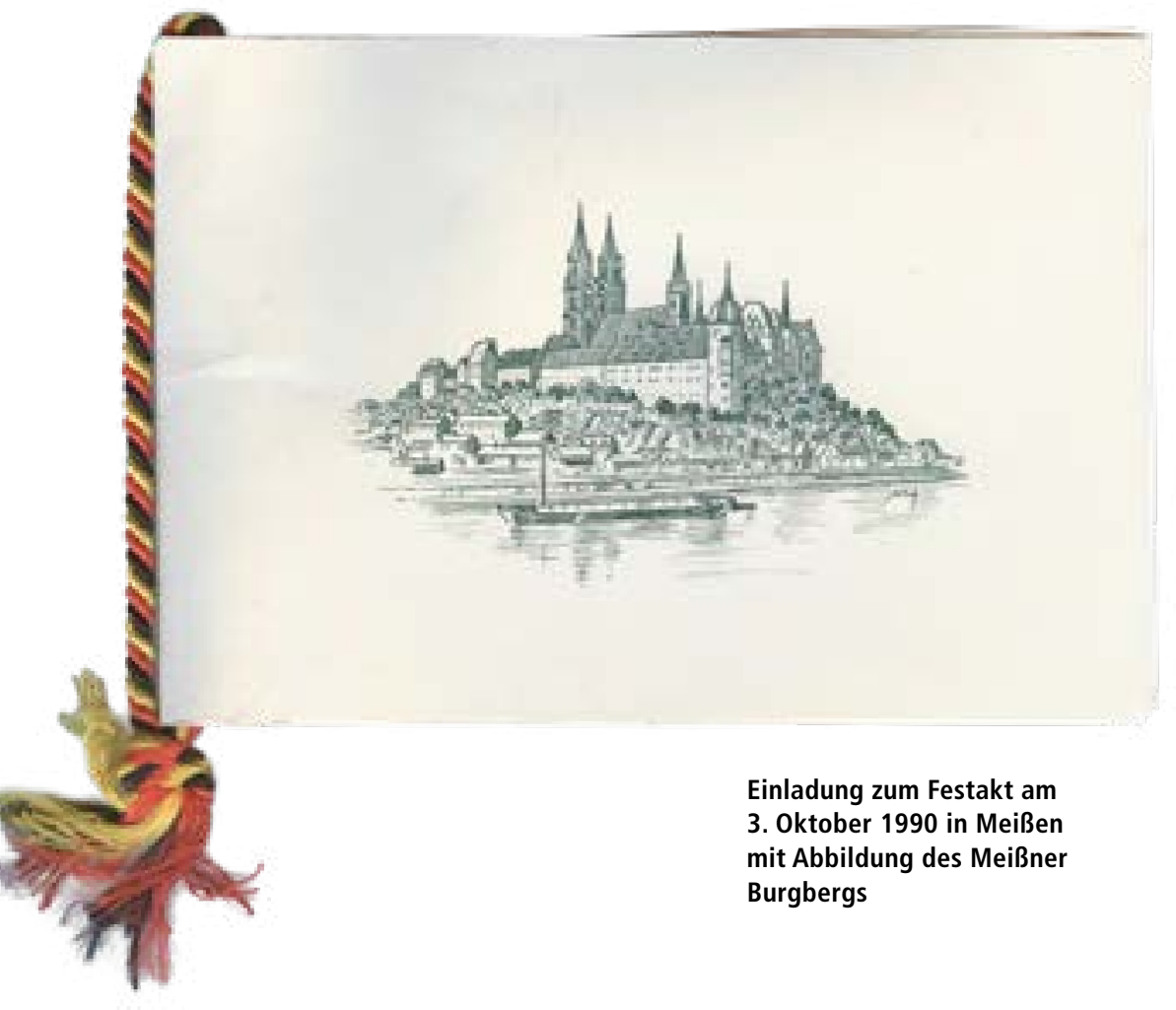

Autos prägten das Straßenbild. Auf den unterschiedlichsten Ebenen, in zahlreichen örtlichen wie überregionalen Arbeitsgruppen und „Runden Tischen“ wurden Überlegungen zur Neugründung Sachsens angestellt.

\section{Initiativen neuer und alter politischer Kräfte zur Länderbildung}

Auch die etablierten politischen Kräfte der DDR, die bislang das zentralistisch-diktatorische System repräsentiert hatten, ignorierten das sächsische Identitätsgefühl nicht, das sich nun wieder artikulieren konnte, und versuchten, auf den fahrenden Zug aufzuspringen. 
Sächsisches Staatsarchiv, Staatsarchiv Leipzig (folgend StA Leipzig), 38212, Niederschrift zur Dienstberatung beim Stellvertreter des Vorsitzenden des Ministerrates für örtliche Staatsorgane, Peter Moreth, am 31. Januar 1990.

2 Vgl. Erich Iltgen, zitiert in Lesch, Markus: Die CDU-Reformer in Sachsen. In: Die Politische Meinung, Heft 297 (1994), S. 38

3 Vgl. Interview mit Erich Iltgen. In: Der Sächsische Landtag. Von der Wende zum Parlament. Sachsen auf dem Weg zur Demokratie. Hrsg. v. Sächsischen Landtag, Dresden 1991, S. 32; Sächsisches Staatsarchiv, Hauptstaatsarchiv Dresden (folgend HStA Dresden), BT/RdB 47121/1, Bl. 239, Bericht des Rates des Bezirkes zur 19. Tagung des Bezirkstages Dresden am 26. April 1990.

4 Vgl. Privatbestand Matthias Rößler, Protokoll der 11. Beratung des Runden Tisches des Bezirkes Dresden am 1. März 1990.

5 Vgl. Suzanne Drehwald/Christoph Jestaedt: Sachsen als Verfassungsstaat. Leipzig 1998 (folgend Drehwald/Jestaedt 1998), S. 73 .

6 Vgl. Interview mit Steffen Heitmann; Hans von Mangoldt: Die Verfassung des Freistaates Sachsen. Entstehung und Gestalt. In: Sächsische Verwaltungsblätter 1 (1993), S. 28; Verfassung des Freistaates Sachsen, BadenBaden 1993, S. 6-7; Drehwald/ Jestaedt 1998, S. 74.

7 Vgl. Heitmann, Steffen: Geschichtliche Entwicklung. In: Christoph Degenhart/Claus Meissner (Hrsg.): Handbuch der Verfassung des Freistaates Sachsen. Stuttgart 1997, S. 43-44 (folgend Heitmann 1997); Privatbestand Klaus-Dieter Scholz, Verfassung des Landes Sachsen. Textentwurf der Gruppe der 20 vom 13. März 1990.

8 Einladung zur Konstituierung des Kuratoriums „Land Sachsen" in Meißen. In: Michael Richter: Die Bildung des Freistaates Sachsen 1989/90. Friedliche Revolution, Föderalisierung, deutsche Einheit. Göttingen 2004 (folgend Richter 2004), Dokument 50.

9 Staatsministerium Baden-Württemberg, Archiv, 0136 Partnerschaft mit Dresden/Sachsen, Schreiben von Michael Kunze an Lothar Späth vom 11. April 1990.
Die neuen politischen Kräfte wehrten sich allerdings gegen Versuche insbesondere bisheriger SED-Funktionäre und Staatsorgane, die Landesbildung an sich $\mathrm{zu}$ ziehen. Auch die Regierung Modrow schrieb sich die Föderalisierung auf ihre Fahne. Ende Januar 1990 wurde die Länderbildung durch den Ministerrat politisch grundsätzlich entschieden. ${ }^{1}$ Am 1. Februar stellte der Rat des Bezirkes Dresden erste Gedanken über die Wiedergeburt Sachsens und Grundzüge einer Landesverfassung vor. Das Ziel des alten Apparates war es, so Erich Iltgen, damals Moderator des Runden Tisches des Bezirkes Dresden, „selbst das Land zu bilden und damit die personellen Strukturen der DDR-Zeit zu konservieren“.2 Der Bezirkstag beschloss die Bildung einer Arbeitsgruppe „Bildung des Landes Sachsen“. Die neuen Kräfte setzten diesen Vorstößen ihrerseits Verfassungsentwürfe entgegen und machten damit ihren Anspruch auf maßgebliche Mitwirkung bei der Bildung des Landes deutlich. Am Runden Tisch des Bezirkes Dresden forderten sie, am Länderbildungsprozess aktiv beteiligt zu werden. ${ }^{3}$ Am 15. Februar 1990 schlug der Dresdner Runde Tisch ein koordiniertes Vorgehen aller drei sächsischen Bezirke für einen gemeinsamen Ausschuss des Landes Sachsen vor. Der Rat des Bezirkes seinerseits beschloss am 28. Februar die ständige Abstimmung und Koordinierung mit den Räten der Bezirke Leipzig und Karl-Marx-Stadt. Am 1. März stimmte der Runde Tisch Dresden der Bildung einer Initiativgruppe zu, die sämtliche Aktivitäten aller Runden Tische und Räte der Bezirke zur Bildung des Landes koordinieren sollte. ${ }^{4}$ Trotzdem blieb die Mehrgleisigkeit erhalten und bestimmte die erste Phase der Landesbildung.

Die neuen politischen Kräfte erkannten, dass sie die Wiedererrichtung Sachsens und die Verfassungsgebung stärker zu ihrer Angelegenheit machen mussten. Angesichts der Aktivitäten des Rates des Bezirkes Dresden zur Ausarbeitung einer sächsischen Verfassung präsentierte der Mitbegründer und Pressesprecher des Dresdner Neuen Forums, Arnold Vaatz, daher Ende März im Namen der Gruppe der 20 einen Verfassungsentwurf. Damit wurde der Versuch des Bezirkstages gekontert, die Wiedergründung des Landes Sachsen als erster in Angriff zu nehmen. ${ }^{5}$ Dieser Entwurf der Gruppe der 20 erschien bereits am 29. und 30. März 1990 in der Tageszeitung „Die Union“. Von nun an verlagerte sich die Verfassungsdiskussion in Richtung der neuen politischen Kräfte.
Freilich war allen Beteiligten klar, dass das von Vaatz erstellte Papier nur ein, wenn auch zentraler, Impuls sein konnte. Für die künftige Arbeit galt es, einen von der breiten Bevölkerung getragenen Verfassungsentwurf $\mathrm{zu}$ erarbeiten. Um dies zu erreichen, wirkten Mitglieder der zeitweiligen Arbeitsgruppe Recht der Dresdner Stadtverordnetenversammlung später in einer paritätisch besetzten Arbeitsgruppe Sachsen/Baden-Württemberg mit, die sich unter dem Vorsitz von Steffen Heitmann in drei Sessionen im Kurort Gohrisch in der Sächsischen Schweiz mit der Formulierung einer Verfassung beschäftigte. Heitmann, Kirchenjurist der sächsischen Landeskirche, fungierte seit Oktober 1989 als juristischer Berater der Gruppe der 20. Die nun von ihm geleitete Arbeitsgruppe war eine unter mehreren Fachgruppen der „Gemischten Kommission Baden-Württemberg/Sachsen", die aufgrund einer Vereinbarung zwischen dem baden-württembergischen Ministerpräsidenten Lothar Späth und den Vorsitzenden der drei sächsischen Bezirke gebildet worden war. Ihr gehörten aber auch Mitglieder der Bürgerbewegungen, neuen Parteien und der Gruppe der 20 an. Hier entstand der am 5. August 1990 vorgestellte „Gohrischer Entwurf”, mit dem der Grundstein für die neue Verfassung Sachsens gelegt wurde. ${ }^{6}$ Es ist wohl richtig, zu sagen, dass die Wurzeln der sächsischen Verfassung vor allem bei der Gruppe der 20 liegen.

\section{Verhinderter Festakt der Räte der Bezirke auf der Albrechtsburg}

Der Konflikt zwischen den Staatsorganen des alten Regimes und Vertretern neuer politischer Gruppen und Parteien sowie der CDU eskalierte, als die Räte der Bezirke Ende April 1990 versuchten, durch einen Festakt auf der Albrechtsburg in Meißen die Bildung eines „Kuratoriums Land Sachsen” zu installieren. Die etablierten Bezirksstaatsorgane wussten nur zu gut um die starke symbolische Bedeutung des Ortes. Zum geplanten feierlichen Charakter der Veranstaltung passte der pathetische Einladungstext: „Das Antlitz Sachsens ist schmerzerfüllt. Aus den Dörfern und Städten unserer Heimat wuchs der Wille zur Veränderung. In der Wiederherstellung des Landes Sachsen liegt Hoffnung und Kraft für morgen. Ihre Mitwirkung im Kuratorium ,Land Sachsen' dient diesem Ziel“". Einladungen gingen an Vertreter der Räte, Bezirkstage, Moderatoren der anderen Runden Tische, Persönlichkeiten des öffentlichen Lebens und 
Chefredakteure von Bezirkszeitungen. Die Journalisten wurden außerdem zu einer Presseinformationsfahrt auf dem historischen Dampfer „Diesbar“ und zu einer internationalen Pressekonferenz eingeladen, bei der sich vor allem die Ratsvorsitzenden als Gesprächspartner präsentieren wollten. ${ }^{9}$ Nach Eingang dieser Einladungen schellten am Dresdner Runden Tisch die Alarmglocken. Die Schreiben waren so abgefasst, dass unklar blieb, ob lediglich das Kuratorium oder eben das Land Sachsen auf diesem Festakt konstituiert werden sollte, und ob die Gäste eine neue Landesverfassung verabschieden wollten.

Nach Meinung von Arnold Vaatz steckte hinter der Einladung, die insbesondere an sächsische Honoratioren ging, die Absicht, sich auf diese Weise zu legitimieren. Bei etlichen von ihnen handelte es sich um Künstler, die bereits Anfang Dezember auf dem Dresdner Theaterplatz demonstriert hatten. Dies sei „exakt der Personenkreis der DDR-Elite“ gewesen, „der nicht diskreditiert war durch Apparate“ und „zu denen der DDR-Bürger aufschaute“. Es handelte sich um Künstler und andere unabhängige Persönlichkeiten, die „erreicht hatten, eine Instanz zu bleiben, auch im Sozialismus". Dabei habe es sich um „bedeutende, unantastbare Persönlichkeiten“ wie Manfred von Ardenne, Kurt Masur oder Theo Adam gehandelt. Dieses „entscheidende Identifikationspotential“ wollten die Räte der Bezirke in erster Reihe der Meißner Veranstaltung platzieren, um „damit der Öffentlichkeit zu suggerieren, jetzt haben die Entscheidungsträger, das intellektuelle Potential des Freistaats Sachsen, diesen Gründungsakt akzeptiert“. Gemäß „mittelalterlicher Methoden“ hätten die Räte damit einen „Huldigungsakt" nach dem Prinzip inszeniert, dass der Landesherr auf die Albrechtsburg kommt, die Untertanen ihm huldigen und damit seine Macht legitimieren. ${ }^{10}$

Aufschlussreich war die geplante Zusammensetzung des „Kuratoriums Land Sachsen“. Die Teilnehmerliste des Bezirkes Karl-Marx-Stadt zeigt ein eklatantes Missverhältnis zwischen den inzwischen per Volkskammerwahl demokratisch legitimierten und marginalisierten Kräften. Neben CDU, SPD, DA, DSU, FDP/ LDPD sollten dem Kuratorium die inzwischen völlig unbedeutenden, teils noch immer der PDS nahestehenden Altparteien und -organisationen DFD, NDPD, DBD, Kulturbund, FDJ, FDGB und VdgB angehören, dazu der Ratsvorsitzende, der Oberbürgermeister von Karl-Marx-Stadt und ein Vertreter der
Bezirksbehörde der Deutschen Volkspolizei. Von den neuen Gruppierungen wurden Grüne Liga, DFP, Grüne Partei, Vereinigte Linke und Neues Forum genannt. Hinzu kamen zwei Vertreter der Kirchen. ${ }^{11}$ Ähnlich sah die geplante Delegation des Bezirkes Dresden aus ${ }^{12}$, so dass es auch hier ein deutliches Übergewicht der marginalisierten und PDS-nahen Kräfte gegeben hätte und die Mehrheitsparteien in eine Minoritätsposition gedrängt worden wären.

Missverständlich und folgenreich war nach dem Treffen am 10. April eine Presseinformation des erweiterten Präsidiums des Bezirkstages Leipzig, wonach „der Regionalausschuß die verfassungsgebende Versammlung für das Land Sachsen sein“ werde ${ }^{13}$. „Die Union“ berichtete daraufhin, dass die Konstituierung eines „Kuratoriums Land Sachsen“ als „verfassungsgebendes Gremium “ vorgesehen sei. ${ }^{14}$ Die Tatsache, dass sich die Nachricht so deuten ließ, als sei die Veranstaltung in Meißen als verfassungsgebende Versammlung konzipiert, weckte auch den Argwohn der Regierungsparteien. Am Rande von Koalitionsverhandlungen in Berlin einigten sie sich, der Einladung am 18. April keine Folge zu leisten, da es "keine demokratische Legitimierung" gab, die Koalitionspartner „den Alleingang eines Landes" ablehnten und „eine Vorreiterrolle der PDS-Funktionäre " nicht hinnähmen. ${ }^{15}$ Parallel dazu setzten in Sachsen die neuen Kräfte um Arnold Vaatz alles daran, sich die mit Hilfe des Dresdner Runden Tisches in Angriff genommene Initiative zur Landesbildung nicht mehr aus der Hand nehmen zu lassen. Es kam zu einem regelrechten Wettrennen mit dem Staatsapparat. Erich Iltgen machte als Vertreter des Runden Tisches im Präsidium des Bezirkstages Druck. Auf sein Drängen wurde hier festgelegt, die Konstituierung des „Kuratoriums Land Sachsen“ nicht in der angedachten Form durchzuführen. Am 14./15. April veröffentlichte „Die Union“ unter dem Titel „Vorschlag zur Länderbildung in der DDR“ eine Erklärung mit Unterschriften aus den Landesvorständen der CDU und des DA sowie aus weiteren Verbänden beider Regierungsparteien. Darin wurde den Räten die Legitimation abgesprochen, Initiativen zur Landesbildung zu ergreifen und diese in die Verantwortung der Regierung gelegt. Eine zügige Länderbildung werde durch Sonderwege wie in Meißen behindert. Schritte zur Länderbildung müssten mit der Regierung abgestimmt sein und von ihr verantwortet werden. Ein Festakt als Start zur Gründung des Landes Sachsen sei der Sache angemes-
10 Arnold Vaatz beim Workshop des Hannah-Arendt-Instituts Dresden am 15. Juni 2002.

11 Hannah-Arendt-Institut Dresden, KA, 06, Teilnehmer des Bezirkes Karl-Marx-Stadt „Kuratorium Land Sachsen“, ohne Datum.

12 Hannah-Arendt-Institut Dresden, KA, 06, Teilnehmer des Bezirkes Dresden „Kuratorium Land Sachsen" vom 3. April 1990.

13 StA Leipzig, BT/RdB, 25795, RdB Leipzig, Vorbereitung Regionalausschuß vom 21. März 1990.

14 Vgl. Die Union, Ausgabe Leipzig vom 12./13. April 1990.

15 Schreiben von Rainer Eppelmann an Wolf-Dieter Beyer vom 9. April 1990. In: Richter 2004, Dokument 43. 
16 „Rottenburger Erklärung“ zur Länderbildung in der DDR vom 6. April 1990. In: Richter 2004, Dokument 39.

17 Erklärung des DA Westsachsen zur Tagung auf der Meißner Albrechtsburg vom April 1990. In: Richter 2004, Dokument 53.

18 Hannah-Arendt-Institut Dresden, KA, 06, Telegramme des Rates des Bezirkes Dresden an Medien und Zeitungen vom 17. April 1990.

19 Vgl. Interview mit Erich Iltgen. In: Sächsischer Landtag (Hrsg.): Der Sächsische Landtag. Von der Wende zum Parlament. Sachsen auf dem Weg zur Demokratie. Dresden 1991, S. 32.

$20 \mathrm{Vgl}$. Interview mit Arnold Vaatz. In: Sächsischer Landtag (Hrsg.): Der Sächsische Landtag. Von der Wende zum Parlament. Sachsen auf dem Weg zur Demokratie. Dresden 1991, S. 47.

21 HStA Dresden, BT/RdB Dresden, 46074, Bl. 4f., Rat des Bezirkes Dresden, Beschluß $105 / 90$ vom 9. Mai 1990, Maßnahmen zur Auswertung der 19. Tagung des Bezirkstages vom 26. April 1990.

22 HStA Dresden, BT/RdB Dresden, 46074, Bl. 107, Stellungnahme des Abg. Dr. Rößler - basisdemokratische Fraktion, auf der 19. Tagung des BT Dresden am 26. April 1990.

23 Hannah-Arendt-Institut Dresden, Iltgen 5, Rat des Bezirkes Dresden. Protokoll der 18. Tagung des Runden Tisches am 3. Mai 1990. sen, wenn er einen demokratischen Prozess einleite und die Öffentlichkeit einbeziehe. Festakte als „Ersatz für Demokratie“ seien „hinreichend bekannte Instrumente stalinistischer Herrschaft “' ${ }^{16}$ In einer vom Pressesprecher des Chemnitzer DA, Wieland Orobko, unterzeichneten Erklärung hieß es, in Meißen solle Sachsen ausgerechnet auf Initiative derer wiederentstehen, die es „im sozialistischen Größenwahn“ in Bezirke zerrissen hätten. Das Volk werde dabei wie 1952 nicht gefragt. Auf der Albrechtsburg solle ein Verfassungsentwurf diskutiert werden, den niemand "außer den einladenden Genossen“ kenne. Der DA empfahl, dass sich diejenigen, die „unter der SED-Fahne die Bezirke ins Chaos geführt" hätten, besser zurückhielten". ${ }^{\text {"17 }}$ Die Proteste, allen voran die gemeinsame Erklärung der an der Regierung beteiligten Parteien, setzten die Räte und Bezirkstage so unter Druck, dass sie die Meißner Tagung absagen mussten. Am Mittag des 17. April 1990 teilte der Rat des Bezirkes Dresden mit, die Informationsfahrt auf dem Dampfer „Diesbar" werde verschoben. ${ }^{18}$ Mit der Verhinderung des Versuchs der Räte, sich in Meißen als führende Kraft der Landesbildung zu profilieren, war es den neuen wie etablierten politischen Kräften, die durch die Volkskammerwahl in die politische Verantwortung gerufen worden waren, gelungen, ihren Anspruch durchsetzten, die Landesbildung selbst in die Hand zu nehmen.

\section{Runde Tische bilden Koordinie- rungsausschuss zur Länderbildung}

Nach der Absage der Meißner Tagung ergriff Erich Iltgen als Moderator des Runden Tisches Ende April im Gegenzug die Initiative zur Gründung eines vor allem von neuen politischen Kräften getragenen Koordinierungsausschusses zur Bildung des Landes Sachsen. ${ }^{19}$ Dieser neue Ausschuss sollte nun die rechtsstaatlichen Grundlagen für die Landesbildung schaffen..$^{20}$ Iltgen forderte die Vertreter der sächsischen Bezirke auf, die Dreigleisigkeit - Rat des Bezirkes, Runder Tisch und Bezirkstag - zu überwinden. Er schlug die Bildung eines gemeinsamen Plenums der drei sächsischen Bezirke vor, das als beschlussfähiges Gremium der öffentlichen Diskussion dienen und die Herausbildung einheitlicher Strukturen des Landes sichern sollte. ${ }^{21}$ Der Dresdner Bezirkstag bestätigte diesen Plan und verpflichtete sich zur Abstimmung aller notwendigen Schritte mit dem Runden Tisch. Allerdings versuchte er, die Arbeit des Gremiums zu kontrollieren und über die Weiterarbeit der am 1. Februar vom Bezirkstag ge-schaffenen Arbeitsgruppe „Bildung des Landes Sachsen“ zu entscheiden. Damit blieb der eigentliche Streitpunkt erhalten, welches Gremium bei der Vorbereitung der Landesgründung, das heißt konkret bei der Einberufung des bezirksübergreifenden Plenums und des Koordinierungsausschusses, die wesentliche Gestaltungskompetenz besaß. Hintergrund der Auseinandersetzungen war die Frage, ob die durch die Volkskammerwahl gestärkten Vertreter der ehemaligen BlockCDU mit ihrem nun bestimmenden Einfluss auf die Politik der Räte oder die neuen politischen Kräfte, die in Dresden zum Teil in der CDU eine neue Heimat gefunden hatten, stärker demokratisch legitimiert waren. Indirekt von der Volkskammerwahl abgeleitete parlamentarisch-demokratische Legitimierung auf Bezirksebene kollidierte mit dem direktdemokratischen Mandat der neuen politischen Kräfte am Runden Tisch. Dem Konflikt der neuen Kräfte mit den PDS-dominierten Staatsorganen folgte nun der mit der dominanten Ost-CDU. Matthias Rößler, damals Entwicklungsingenieur an der TU Dresden und Mitglied des Landesvorstandes des DA, wies im Dresdner Bezirkstag für die Basisdemokratische Fraktion ausdrücklich darauf hin, dass das Plenum kein Gremium des Rates des Bezirkes darstelle. Die Initiative des Runden Tisches dürfe von diesem nicht zur Legitimierung missbraucht werden. ${ }^{22}$

Angesichts des Beharrungsvermögens der Räte forcierten die neuen politischen Kräfte die Länderbildung. Bis Anfang Mai trug eine Arbeitsgruppe des Runden Tisches alle Vorschläge der beteiligten Parteien zur Länderbildung zusammen und legte einen Rahmenvorschlag für die Zusammenarbeit mit den Bezirken Leipzig und Chemnitz vor. Darin wurde an die Vorschläge Iltgens angeknüpft und die Bildung eines Vorparlamentarischen Ausschusses und des von den drei Bezirken paritätisch zu bildenden Koordinierungsausschusses geplant, zu dessen Vorsitzendem der Runde Tisch des Bezirkes Dresden am 3. Mai einstimmig Arnold Vaatz wählte. ${ }^{23}$ Als der für die Bildung der Landesstrukturen zuständige Stellvertreter des Regierungsbeauftragten für den Bezirk Dresden hatte Vaatz die auch von den Bezirken Chemnitz und Leipzig in die Dresdner Kompetenz übertragenen Aktivitäten zu leiten. Der Koordinierungsausschuss, der am 25. Mai erstmals tagte, prägte in der Folge die Geschicke des Landes bis zu den ersten Landtagswahlen wie kaum eine zweite 
Institution und übernahm in der Folgezeit die Leitfunktion bei der Landesbildung. Dabei verstand er sich als ein durch Akklamation eingesetztes Gremium, dessen Ziel es war, sich möglichst bald selbst überflüssig zu machen. ${ }^{24}$

\section{Haltung der DDR-Regierung zur Länderbildung}

Die sächsische Entwicklung wurde maßgeblich von Entscheidungen der DDR-Regierung beeinflusst. Rat des Bezirkes, Bezirkstag und Runde Tische waren davon gleichermaßen betroffen. Die Regierung de Maizière sah sich angesichts des fortgeschrittenen Zerfalls staatlicher Autorität und der sich abzeichnenden Länderbildung nach der Regierungsübernahme im April 1990 veranlasst, schnell über die Zukunft der Bezirksgremien zu entscheiden. Gegenüber den Vorsitzenden der Räte der Bezirke betonte Lothar de Maizière am 23. April die Bedeutung der Aufrechterhaltung der staatlichen Strukturen, die nun die am 18. März geschaffenen Mehrheitsverhältnisse widerspiegeln müssten. Er informierte darüber, dass zahlreiche Bezirke nicht mehr handlungsfähig seien und die Regierung eine Beendigung der Arbeit der Bezirkstage für Ende Mai 1990 vorsehe. Die Räte würden von diesem Zeitpunkt an nur noch als Auftragsverwaltungen der Regierung tätig werden. ${ }^{25}$ Der Ministerrat bevorzugte eine Anbindung an die Regierung, weil diese, auch im Gegensatz zu den Runden Tischen der Bezirke, als einzige durch freie Wahlen legitimiert war, und folgte daher zugleich der Auffassung, dass das Wirken der Runden Tische durch die Wahlen überflüssig geworden sei.

Angesichts der Dringlichkeit der Länderneubildung favorisierte die Regierung de Maizière ein Gesetzesvorhaben, das sich an den alten Ländern der DDR orientierte. Am 2. Mai bestätigte der Ministerrat seine Entscheidung, die Verwaltungsorgane der Bezirke bis zur Länderbildung als Auftragsverwaltungen $\mathrm{zu}$ erhalten, und beschloss, bis zum 10. Juni Regierungsbevollmächtigte in den Bezirken einzusetzen. Diese sollten von den Parteien nominiert werden, die dort am 18. März die meisten Stimmen erhalten hatten. In Sachsen war dies in allen Bezirken die CDU. Am 17. Mai beschloss die Volkskammer offiziell, die Legislaturperiode der Bezirkstage mit Wirkung vom 31. Mai zu beenden und beauftragte den Ministerpräsidenten mit der Einsetzung der Regierungsbevollmächtigten. Ihnen wurden beratende Gremien an die Seite gestellt, die sich aus den am 18. März gewähl- ten Volkskammerabgeordneten der jeweiligen Bezirkes zusammensetzten. Die Räte der Bezirke wurden in Bezirksverwaltungen unter Leitung der Regierungsbevollmächtigten umgewandelt. ${ }^{26}$ Beschlüsse noch bestehender Runder Tische wurden für das exekutive Handeln der Bezirksverwaltungen als nicht mehr verbindlich bezeichnet. ${ }^{27}$ Am 29. Mai stellte der Dresdner Bezirkstag seine Arbeit ein. ${ }^{28}$

Die Entscheidung des Ministerrates stieß beim Runden Tisch des Bezirkes Dresden auf Ablehnung. Hier waren vor allem die neuen politischen Kräfte nicht ohne weiteres bereit, die soeben gewonnene Mitsprache gleich wieder aufzugeben. Vor allem in die CDU eingetretene Bürgerrechtler fürchteten, dass der alte Machtapparat, repräsentiert durch die jetzt „Bezirksverwaltungen“ genannten Räte, bis zu den Landtagswahlen erhalten bleiben würde ${ }^{29}$, und hielten es für notwendig, bei der Kontrolle der Bezirksverwaltungen und der Bildung des Landes Sachsen weiterhin eine wesentliche Rolle zu spielen. Die im revolutionären Prozess von breiten Bevölkerungsteilen direkt-demokratisch legitimierten politischen Neulinge trauten den nun in die erste Reihe vorgerückten früheren Vertretern der Block-CDU nicht recht. Umgekehrt zogen diese die direkt-demokratische Legitimierung der politischen Seiteneinsteiger in Frage. Dieser politische Streit wurde innerhalb der sächsischen CDU zwischen den sogenannten „Reformern” und Vertretern der früheren Block-CDU ausgetragen. ${ }^{30}$ Der Konflikt war Ausdruck des diffizilen, mehrschichtigen Überganges von direktdemokratischen zu parlamentarisch-demokratisch legitimierten Vertretungskörperschaften.

Auf der 20. Sitzung des Runden Tisches am 31. Mai 1990 reagierten die Teilnehmer abermals mit Unwillen auf den Ministerratsbeschluss, den Runden Tischen der Bezirke jegliche Kompetenz gegenüber den Verwaltungsorganen abzusprechen. Dennoch wurde beschlossen, dass der Runde Tisch des Bezirkes Dresden seine Arbeit zur Länderbildung beenden solle. ${ }^{31}$ In Anlehnung an den Ministerratsbeschluss „Zur Sicherung der Regierungsfähigkeit in den Bezirken bis zur Bildung funktionsfähiger Länder” wurde außerdem der Vorschlag erörtert, den geplanten „Vorparlamentarischen Ausschuß“ in „Regionalausschuß Land Sachsen" umzubenennen, um deutlich zu machen, dass eine Zuständigkeitskonkurrenz mit den Regierungsbeauftragten bzw. den diesem zugeordneten sächsischen Volkskammerabgeordneten nicht gegeben sei. ${ }^{32}$

Am 5. Juni 1990 erließ der Ministerrat Richtlinien über Stellung, Aufgaben und Befugnisse
24 Vgl. Arnold Vaatz: Die friedliche Revolution war ein guter Anfang. In: Frankfurter Allgemeine Zeitung vom 19. Mai 1994.

25 LandeshauptarchivSachsen-Anhalt (folgend LHASA), 30015 , RdB Magdeburg, Protokoll der Tagung des Runden Tisches Sachsen-Anhalt (Regionalausschuß), Halle/S., 24. April 1990.

26 Brandenburgisches Landeshauptarchiv, A/4993, Beschluß der Volkskammer der DDR zur Beendigung der Legislaturperiode der Bezirkstage vom 17. Mai 1990.

27 Bundesarchiv Berlin (folgend BArch Berlin), C-20, I/3-2947, Bl. 22-24, Beschluß des Ministerrates der DDR 5/2/90 vom 2. Mai 1990.

28 HStA Dresden, BT/RdB Dresden, 46075, Protokoll 20. Tagung Bezirkstag Dresden vom 29. Mai 1990.

29 Vgl. Erich Iltgen: Neue Politik für ein altes Land. Sachsen und die Demokratisierung der ehemaligen DDR. In: Uwe Thaysen/Hans Michael Kloth (Hrsg.): Wandel durch Repräsentation - Repräsentation im Wandel. Entstehung und Ausformung der parlamentarischen Demokratie in Ungarn, Polen, der Tschechoslowakei und der ehemaligen DDR. Baden-Baden 1992, S. 155.

30 Privatbestand Matthias Rößler, Protokoll der 19. Sitzung des Runden Tisches des Bezirkes Dresden am 17. Mai 1990; Ute Schmidt: Von der Blockpartei zur Volkspartei? Die Ost-CDU im Umbruch 1989-1994. Opladen 1997, S. 159-177.

31 Vgl. Sächsischer Landtag (Hrsg.): Der Sächsische Landtag. Von der Wende zum Parlament. Sachsen auf dem Weg zur Demokratie. Dresden 1991, S. 43 .

32 Privatbestand Matthias Rößler, Protokoll 20. Sitzung des Runden Tisches des Bezirkes Dresden am 31. Juni 1990. 
33 LHASA, 30015, RdB Magdeburg, Verfügung des Ministerpräsidenten der DDR vom 5. Juni 1990, Regelungen über Stellung, Aufgaben und Befugnisse der Regierungsbevollmächtigten, vgl. Interview mit Erich Iltgen. In: Der Sächsische Landtag, S. 31.

34 BArch Berlin, C-20, I/3-2981, Bl.1f., Beschluß des Ministerrates der DDR 10/26.a/90 vom 6. Juni 1990 .

$35 \mathrm{Vgl}$. Interview mit Arnold Vaatz. In: Sächsischer Landtag (Hrsg.): Der Sächsische Landtag. Von der Wende zum Parlament. Sachsen auf dem Weg zur Demokratie. Dresden 1991, S. 48 .

36 Interview mit Erich Iltgen. In: Sächsischer Landtag (Hrsg.): Der Sächsische Landtag. Von der Wende zum Parlament. Sachsen auf dem Weg zur Demokratie. Dresden 1991, S. 32.

37 Vgl. Interview mit Erich Iltgen. In: Union vom 12. Juli 1990.

38 Hannah-Arendt-Institut Dresden, Iltgen 3, Schriftwechsel Sächsisches Forum, Anlage (unredigierte Bandaufzeichnung) zur Niederschrift der 2. Öffentlichen Beratung des Sächsischen Forums vom 23. August 1990.

39 GBl. DDR 1990, I, 51, S. 955.

40 BArch Berlin, C-20, I/3-3054, Bl. 131, 27. Sitzung des Ministerrates der DDR am 29. August 1990.

41 BArch Berlin, C-20, I/3-3058, Bl. 34-41, Protokoll der 29. Sitzung des Ministerrates der DDR am 5. September 1990; Mecklenburgisches Landeshauptarchiv Schwerin, RdB Schwerin, Z 123/91 37922, Ministerrat der DDR, Der Ministerpräsident, Regelungen zu Stellung, Aufgaben und Befugnissen der Landessprecher als Landesbevollmächtigte, gez. L. de Maizière, vom 17. September 1990.

42 Interview mit Arnold Vaatz. In: Sächsischer Landtag (Hrsg.): Der Sächsische Landtag. Von der Wende zum Parlament. Sachsen auf dem Weg zur Demokratie. Dresden 1991, S. 49.

43 BGBl. II, S. $885 \mathrm{ff}$. der Regierungsbevollmächtigten ${ }^{33}$ und legte am 6. Juni die personelle Besetzung dieser Funktionen fest. $^{34}$ Mit der Einsetzung des Regierungsbeauftragten für den Bezirk Dresden, des Diplom-Staatswissenschaftlers Siegfried Ballschuh (CDU), der im Rat des Bezirkes Dresden Abteilungsleiter für Wohnungspolitik und -wirtschaft gewesen war, stellte sich die Frage, ob dieser den direkt-demokratisch legitimierten Koordinierungsausschuss akzeptieren oder auflösen würde. Die Entscheidung der Regierung de Maizière richtete sich zunächst gegen den Koordinierungsausschuss. ${ }^{35}$ Eine Auflösung konnte jedoch verhindert werden, da es gelang, die hohe politische Akzeptanz des Ausschusses zu verdeutlichen. Hinzu kam die starke Stellung des Runden Tisches des Bezirkes Dresden, der sich für den Erhalt des Ausschusses einsetzte. Angesichts der konkreten Machtverhältnisse kam Ballschuh nicht umhin, den Koordinierungsausschuss an sein Amt anzubinden. Die Entscheidung, durch die sich die Lage in Sachsen erheblich von der in anderen Teilen der DDR unterschied, bedeutete einen großen Erfolg für die neuen politischen Kräfte. Diese konnten ein Feld besetzen, das bislang eine Domäne der Verwaltungsorgane der Räte der Bezirke gewesen war. Am 14. Juni ernannte Ballschuh Arnold Vaatz zu seinem Stellvertreter mit Zuständigkeit für die Ressorts Verwaltung, Organisation, internationale Fragen und Landesgesetzgebung. Damit erhielt der im Januar 1990 in die CDU eingetretene Bürgerrechtler umfangreiche Vollmachten und Kompetenzen.

Trotz der engen Kooperation mit dem Koordinierungsausschuss stand der Runde Tisch des Bezirkes Dresden der Machtkonzentration bei den Regierungsbevollmächtigten in den Bezirken skeptisch gegenüber. Die den Koordinierungsausschuss gemeinsam stützenden neuen Parteien SPD, DA, DFP und DSU sowie die neuen Kräfte um Vaatz in der CDU meinten weiterhin, die Entwicklung in den Regionen müsse durch regionale Gremien direkt-demokratisch legitimierter Kräfte weiter begleitet werden. ${ }^{36}$ Ungeachtet der Entscheidung des Ministerrates über das Ende des Einflusses direkt-demokratischer Gremien sprach sich der Runde Tisch des Bezirkes daher in seiner 22. Sitzung am 28. Juni 1990 gegen den Vorschlag aus, seine Tätigkeit hinsichtlich der Bildung des Landes Sachsen einzustellen und sich aufzulösen. Der Runde Tisch lehnte auch die Bildung eines Regionalausschusses ab, der als Vertretung der Öffentlichkeit die Arbeit des Regierungsbevollmächtigten begleiten sollte. Erich Iltgen begründete die Ablehnung damit, dieser sei in seiner Wirksamkeit auf den Bezirk Dresden beschränkt. Mit Blick auf das künftige Land Sachsen sei jedoch ein erweitertes, gemeinsames Forum aller drei Bezirke sinnvoll und geboten. Außerdem äußerte er die Befürchtung, die den Regierungsbevollmächtigten zugeordneten Regionalausschüsse der Bezirke könnten von den Mehrheitsparteien als rein repräsentative, quasi-demokratische Garnierung missbraucht werden. Ein solches Gremium gewährleiste nicht das Maß an Öffentlichkeit, das vom Runden Tisch gewünscht werde. Der Runde Tisch des Bezirkes Dresden folgte vielmehr Iltgens Vorschlag, seine Verantwortung bis zur Bildung eines Forums für alle sächsischen Bezirke weiter wahrzunehmen. Iltgen bezeichnete zwar das Anliegen, die Arbeit der Runden Tische zu beenden, als grundsätzlich berechtigt, verwies aber darauf, dass die Bildung des Landes Sachsen aus Sicht des Runden Tisches noch keinesfalls befriedigend gelöst sei. ${ }^{37}$ Erst nachdem sichergestellt war, dass die neuen politischen Kräfte entscheidende Positionen in den Landesministerien und -verwaltungen inne haben würden und die Arbeitsfähigkeit des Koordinierungsausschusses gesichert war, löste der Runde Tisch sich auf. Er beschloss am 12. Juli einstimmig die Bildung eines Sächsischen Forums, in dem die Runden Tische der Bezirke ihre Arbeit auf Landesebene fortsetzten.

\section{Sächsisches Forum und Koordinierungsausschuss}

Das Sächsische Forum bestand aus Vertretern aller Parteien und neuen Gruppierungen sowie aus Volkskammerabgeordneten und Vertretern der Kreise, die Teil des Landes Sachsen werden wollten. Seine Hauptaufgabe lag in der öffentlichen Information über den Stand der Landesbildung. Dazu wurden interessierte Bürger in die Diskussion einbezogen, um so den demokratischen Charakter des Prozesses zu unterstreichen. Allerdings hatten Anregungen des Sächsischen Forums an den Koordinierungsausschuss nur empfehlenden Charakter. So blieb die offizielle Legitimierung des Regierungsbevollmächtigten und seines Stellvertreters für die Landesbildung durch die Regierung der DDR formal erhalten. Auf der ersten öffentlichen Beratung des Sächsischen Forums am 26. Juli wurde dem Forum die Aufgabe zugewiesen, die Öffentlichkeitsarbeit für den Koordinierungsausschuss zur Bildung des Landes Sachsen zu übernehmen und allen Parteien und Gruppen 
eine Plattform zu bieten, zudem noch vorhandene Strukturschwächen der neuen parlamentarischen Demokratie auszugleichen und „Brücke von der Öffentlichkeit zum Parlament“ zu sein. Auf der 2. Beratung des Sächsischen Forums am 23. August stellte Heitmann den inzwischen fertiggestellten „Gohrischen Entwurf " einer sächsischen Verfassung vor. ${ }^{38}$ Damit hatten sich die neuen politischen Kräfte auch bei der Gestaltung der künftigen Verfassung gegen die Initiativen der Räte der Bezirke behaupten können.

Nachdem die Volkskammer am 22. Juli 1990 das Ländereinführungsgesetz ${ }^{39}$ verabschiedet hatte, das die Bildung der Länder zum 14. Oktober 1990 vorsah, setzte der Ministerrat Anfang September Landessprecher ein, die im Ministerrat als Interessenvertreter der künftigen Länder mit beratender Stimme mitarbeiteten. ${ }^{40}$ Der bisherige Regierungsbevollmächtigte für den Bezirk Leipzig, der frühere Funktionär der CDU und Vertraute de Maizières, Rudolf Krause, wurde Landessprecher von Sachsen. Die Landessprecher waren gegenüber dem Ministerrat für alle mit der Länderbildung zusammenhängenden Aufgaben verantwortlich und dienten bis dahin als Verbindungsstellen zwischen der Regierung und den Gebietskörperschaften. Sie hatten die Tätigkeit der Regierungsbevollmächtigten und der Bezirksverwaltungsbehörden zu organisieren und die Bildung funktionsfähiger Landesregierungen einschließlich der Verwaltungsorganisation der Länder, ihrer personellen Besetzung und räumlichen Ansiedlung sowie die Erarbeitung von Verfassungs- und anderen Gesetzesentwürfen zu organisieren. Dazu wurden ihnen Beauftragte für die Bildung der künftigen Ministerien auf Länderebene zugeordnet. ${ }^{41}$ Die Einsetzung des Landessprechers beeinflusste auch die Arbeit des Koordinierungsausschusses. Vaatz bezeichnete den Ausschuss als ausführendes Organ der Bezirksverwaltungen und erreichte, dass der Landessprecher den Ausschuss in die Arbeit seines Amtes einband und ihn durch die Übertragung von Verwaltungsund Weisungsbefugnis gegenüber den Bezirksverwaltungsbehörden sogar erheblich aufwertete. ${ }^{42}$ Der Koordinierungsausschuss verfügte nun über eine wechselnde Anzahl von Stäben, die sich jeweils mit einzelnen Problemen wie der Vorgestaltung von Ministerien, der Sichtung des Landesvermögens oder der Lösung von Raumfragen beschäftigten. Die durch den Koordinierungsausschuss entworfenen Strukturen dienten als Grundlage für den vor allem mit Hilfe der Länder Baden-Württemberg und Bayern betriebenen Aufbau der Regierungsministerien, wobei sich die enge Kooperation zwischen Angehörigen des Koordinierungsausschusses und der im September 1990 eingerichteten „Clearingstelle“ der Bundesregierung bewährte. Es war eine Besonderheit der Friedlichen Revolution, dass in ihrer letzten, strukturverändernden Phase, in der die Forderungen der Demonstranten vom Herbst 1989 und Winter 1989/90 praktisch umgesetzt wurden, bundesdeutsche Funktionseliten von der veränderungswilligen DDR-Bevölkerung in die Pflicht genommen wurden. Ihnen kam die diffizile Aufgabe der Umsetzung der revolutionären Forderungen nach einer tiefgreifenden Strukturveränderung aller Bereiche von Politik, Wirtschaft, Finanzen, Sozialem, Rechtssystem usw. im Rahmen und im Prozess der Herstellung der staatlichen Einheit Deutschlands zu.

\section{Die Gründung des Freistaates Sachsen}

Der Einigungsvertrag in der Fassung vom 23. September $1990^{43}$ verlegte den im Ländereinführungsgesetz vorgesehenen Zeitpunkt der Neubildung der Länder vom 14. auf den 3. Oktober 1990, den Tag des Beitritts der DDR zur Bundesrepublik. Das Land Sachsen wurde daher bereits an diesem Tag mit einem Festakt auf der Meißener Albrechtsburg wiedergegründet, allerdings stand es bis zur

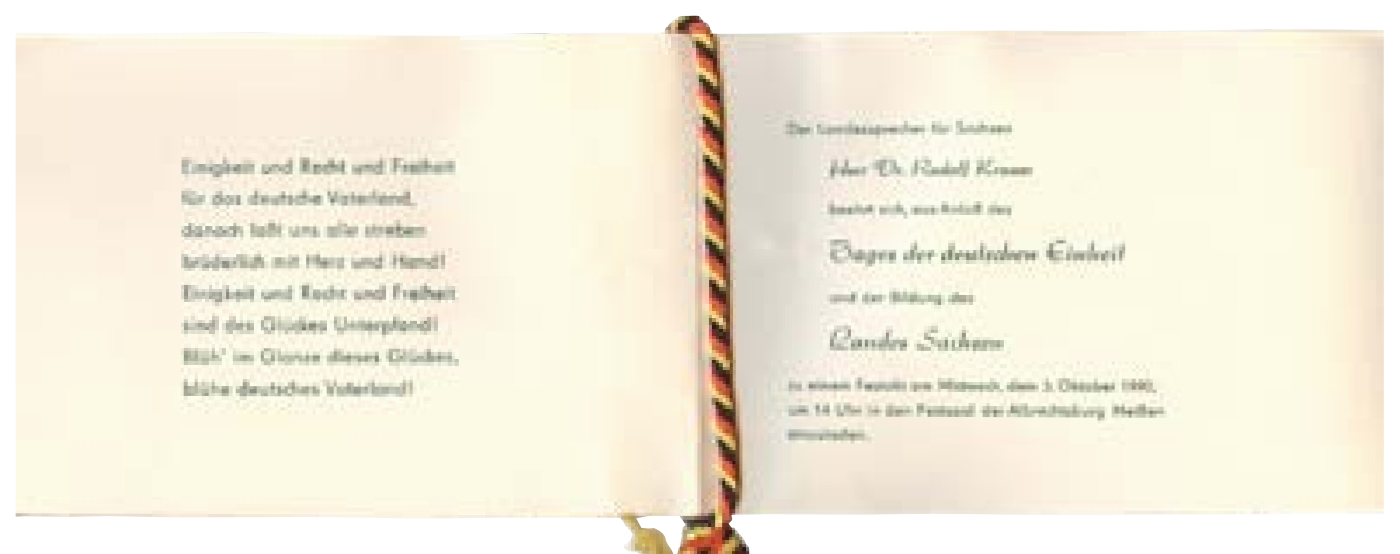


Festversammlung am 3. Oktober 1990 in der Hofstube der Meißner Albrechtsburg

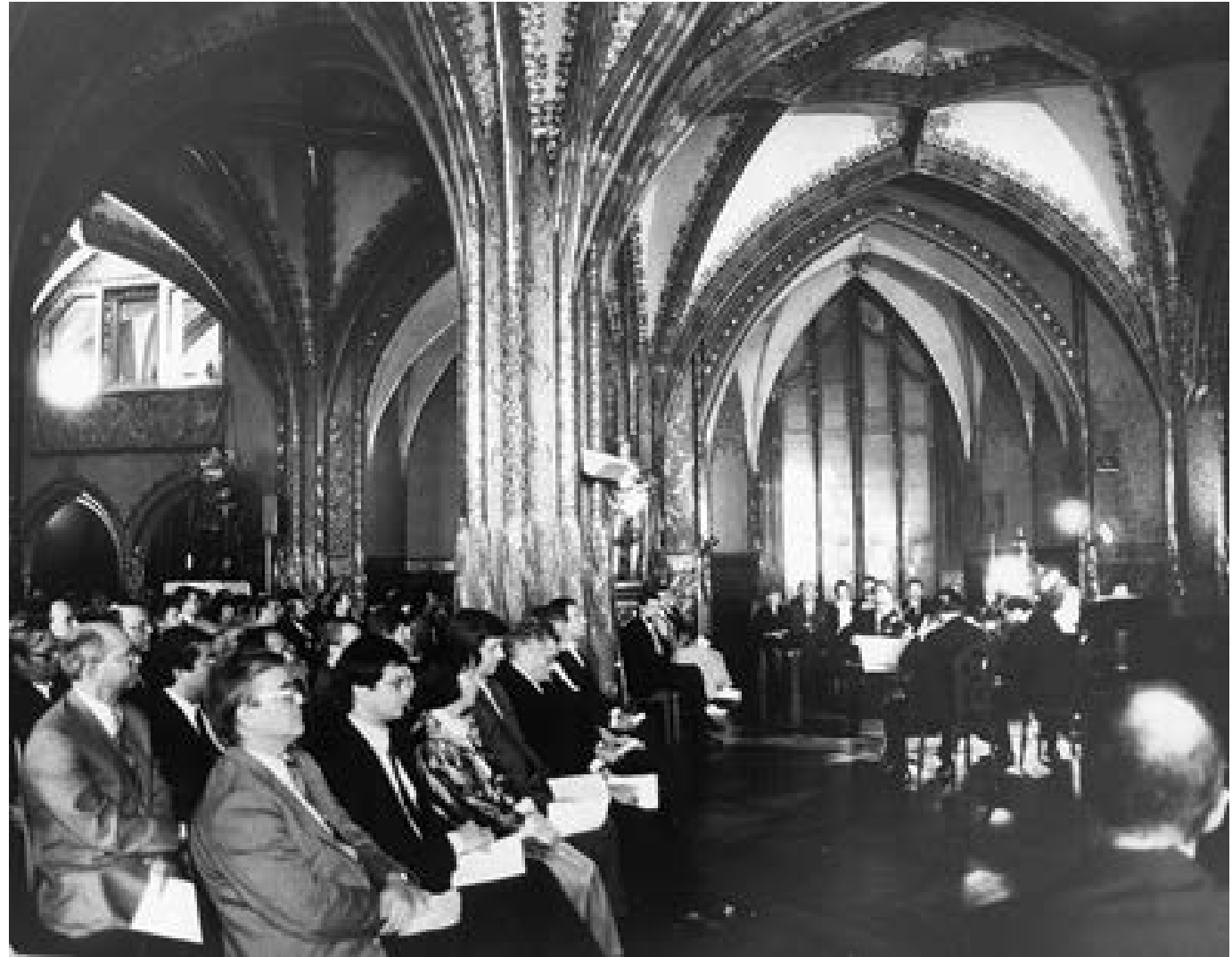

Landtagswahl und der Bildung einer Landesregierung unter der Hoheit des Bundesinnenministeriums. Im Beisein von Vertretern aus Politik und Gesellschaft sowie des früheren Herrscherhauses Wettin gab der Landeshistoriker Karlheinz Blaschke einen historischen Abriss der Geschichte und würdigte dabei auch die Rolle der Albrechtsburg. Es gebe keinen besseren Platz, einen Festakt zur Neubildung des Landes Sachsen zu wählen, als die-

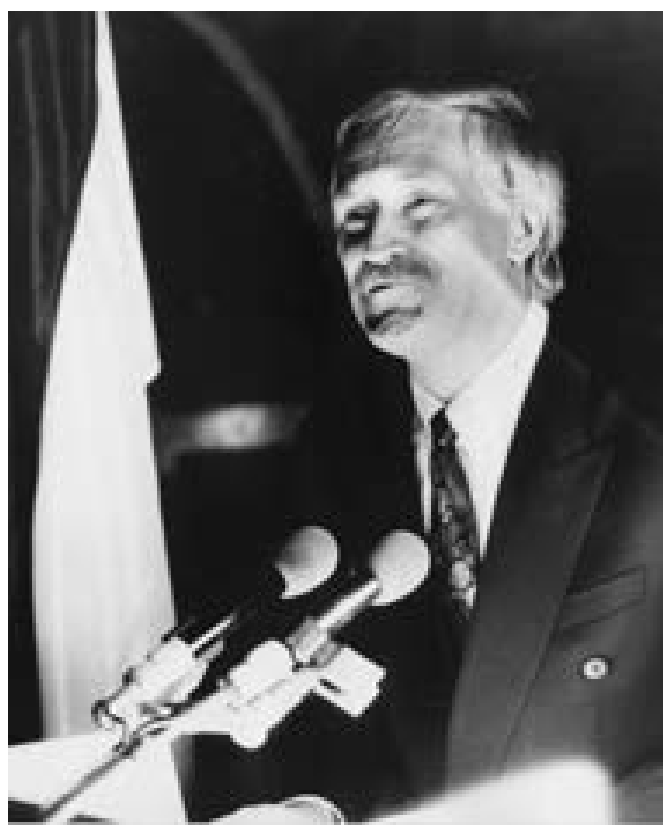

sen Platz, den Burgberg zu Meißen. Hier sei im Jahre 929 die Herrschaft des deutschen Königs errichtet worden, und von diesem Tage an habe die sächsische Geschichte ihren Anfang genommen. Es sei ein Zeichen für das Traditionsbewusstsein der Kräfte, die das Land Sachsen bildeten, dass sie auf die Geschichte zurückblickten. Sie zeigten damit ihr Bewusstsein, dass „auch jedes Bauen in die Zukunft hinein den Mutterboden einer Verwurzelung, einer Tradition braucht, auf dem man fest stehen kann". Auch Rudolf Krause und Erich Iltgen wandten sich in feierlichen Ansprachen an die geladenen Gäste. Krause sprach von einem historischen Tag im Leben des deutschen Volkes und des Landes Sachsen sowie von der Pflicht, „große Achtung gegenüber unseren jungen Menschen zu empfinden, die mutig und entschlossen für unser neues, befreites Leben“ eingetreten seien. Der Beitrag der Bürgerbewegungen dürfe niemals vergessen werden. Er dankte den Partnern aus Baden-Württemberg und Bayern für ihre Hilfe beim Übergang zu neuen Verwaltungsstrukturen. Sein diesbezüglicher Dank galt aber auch den Mitarbeitern der Bezirksverwaltungsbehörden. ${ }^{44}$

Territorial setzte sich das neue Land Sachsen aus den Bezirken Dresden, Chemnitz und Leipzig ohne die Kreise Altenburg und Schmölln zuzüglich der Kreise Hoyerswerda und Weißwasser zusammen. Mit dem Bezirk 


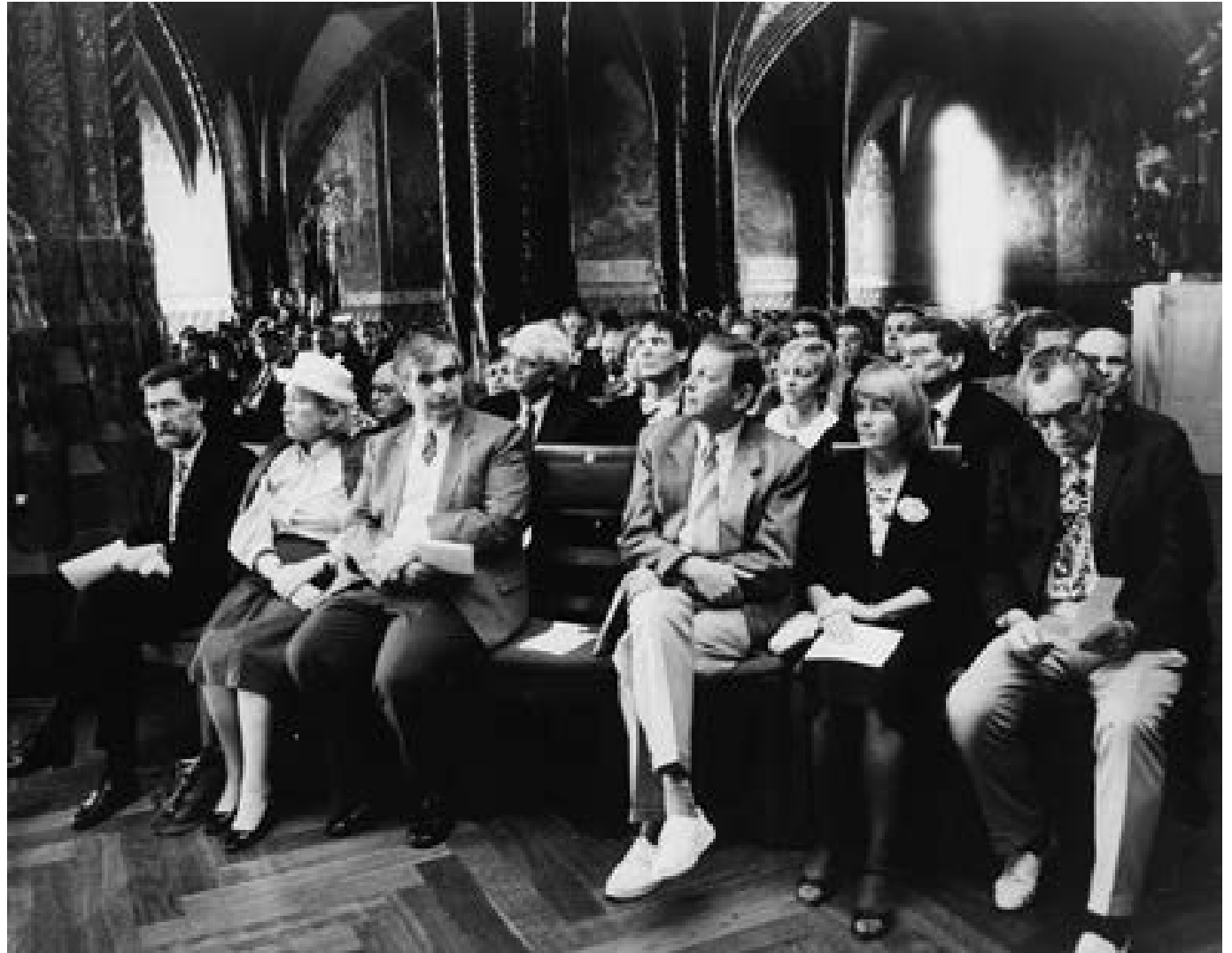

Leipzig gelangten auch die Kreise Delitzsch, Eilenburg und Torgau, die seit 1815 zur preuBischen Provinz Sachsen gehört hatten, wieder nach Sachsen.

Am 14. Oktober 1990 folgte die Wahl des Landtages. Auf dessen konstituierender Sitzung am 27. Oktober wurden Kurt Biedenkopf zum Ministerpräsidenten und Erich Iltgen zum Landtagspräsidenten gewählt. In dieser Sitzung wurde die Bezeichnung „Freistaat Sachsen" festgelegt.

Ein am selben Tag beschlossenes „Vorschaltgeset $z^{“ 45}$ schuf den rechtlichen Rahmen für die Arbeit der staatlichen Organe bis zum Inkrafttreten der zu diesem Zeitpunkt noch nicht fertiggestellten sächsischen Verfassung. Nach der Veröffentlichung des „Gohrischen Entwurfs“46 am 23. August waren zahlreiche Stellungnahmen eingegangen. Daraufhin wurde der Entwurf im Oktober 1990 von Mitgliedern der zuständigen Arbeitsgruppe wiederum in Gohrisch einer Überarbeitung unterzogen. Am 23. Oktober 1990 konnte das Ergebnis, der „Zweite Gohrische Entwurf“, der Öffentlichkeit präsentiert werden. Insgesamt lagen dem Sächsischen Landtag bei seinen nun beginnenden etwa eineinhalbjährigen Verfassungsberatungen drei verschiedene Entwürfe vor. Unter Federführung des Verfassungs- und Rechtsausschusses wurden diese bis zum Mai 1991 diskutiert. Im Juni 1991 wurden die
Arbeitsergebnisse veröffentlicht und der Bevölkerung Gelegenheit zur Stellungnahme gegeben. Daraufhin gingen insgesamt ca. 1.300 Zuschriften ein. Es schlossen sich weitere Ausschussberatungen an, die sich bis in den April 1992 erstreckten. Am 27. Mai 1992 wurde die Verfassung des Freistaates Sachsen schließlich verabschiedet. Sie wurde am 5. Juni 1992 verkündet und trat einen Tag später in $\mathrm{Kraft}^{4}{ }^{47}$ In ihrer Präambel heißt es, dass sich „das Volk im Freistaat Sachsen dank der friedlichen Revolution des Oktober 1989 diese Verfassung gegeben" habe.

Die Meißner Albrechtsburg als Ort der Herausbildung sächsischer Staatlichkeit spielte bei den revolutionären Auseinandersetzungen um die Neubildung Sachsens eine wichtige symbolische Rolle. Mehr als in anderen Regionen der DDR kämpften in Sachsen und insbesondere im Bezirk Dresden Kräfte des untergehenden Regimes und Akteure neuer politischer Bewegungen erbittert um Dominanz im Prozess der Länderbildung. Die Auseinandersetzungen um staatliche Strukturen und personelle Besetzungen stellten eine Fortsetzung des im Herbst 1989 begonnenen revolutionären Prozesses dar. Die Wiedervereinigung Deutschlands und die Neubildung Sachsens am 3. Oktober 1990 waren Abschluss und Höhepunkte der Friedlichen Revolution in Sachsen.
Unter den Teilnehmern befand sich auch Albert Prinz von Sachsen Herzog zu Sachsen (1934-2012), ein Enkel des letzten sächsischen Königs.
44 Reden auf der Meißener Albrechtsburg zum Tag der deutschen Einheit und zur Bildung des Landes Sachsen. In: Richter 2004, Dokument 151. Die Festrede von Karlheinz Blaschke ist wiederabgedruckt in diesem Band.

45 Gesetz zur Herstellung der Arbeitsfähigkeit des Sächsischen Landtages und der Sächsischen Landesregierung (Vorschaltgesetz) vom 27. Oktober 1990. In: SächsGVBl. S. 1.

46 Verfassung des Freistaates Sachsen. Gohrischer Entwurf, überarbeitete Fassung. Dresden 1990.

47 SächsGVBl., S. 243ff., vgl. Heitmann 1997, S. 42

\section{Autor}

Dr. Michael Richter

Sorbisches Institut

Bahnhofstraße 2

02625 Bautzen 